\title{
Protée
}

\section{Empreinte et réincarnation}

\section{Benoît Mauchamp}

Volume 39, numéro 2, automne 2011

URI : https://id.erudit.org/iderudit/1007172ar

DOI : https://doi.org/10.7202/1007172ar

Aller au sommaire du numéro

\section{Éditeur(s)}

Département des arts et lettres - Université du Québec à Chicoutimi

\section{ISSN}

0300-3523 (imprimé)

1708-2307 (numérique)

Découvrir la revue

\section{Citer cet article}

Mauchamp, B. (2011). Empreinte et réincarnation. Protée, 39(2), 95-104. https://doi.org/10.7202/1007172ar

\section{Résumé de l'article}

L'article considère le concept bouddhiste de réincarnation au regard des éléments d'une sémiotique de l'empreinte telle qu'elle est proposée par Jacques Fontanille dans son livre Soma et Séma (2004). Après avoir dégagé les

caractéristiques communes de la réincarnation et d'une théorie de l'empreinte, qui toutes deux allient notamment présence et absence, immanence et transcendance, rupture et continuité, le propos s'efforce d'observer plus concrètement ces phénomènes à travers l'étude d'objets filmiques. Les deux films considérés - une fiction populaire de Bertolucci, Little Buddha (1993), et un documentaire de Nati Baratz, Unmistaken Child (2008) - possèdent une intrigue similaire invitant à étudier, sur le plan de l'expression, comment l'empreinte d'un être éveillé peut s'inscrire dans le substrat signifiant du corps et, sur le plan du contenu, la quête d'un moine pour la nouvelle manifestation de son maître décédé.
Ce document est protégé par la loi sur le droit d'auteur. L'utilisation des services d’Érudit (y compris la reproduction) est assujettie à sa politique d'utilisation que vous pouvez consulter en ligne.

https://apropos.erudit.org/fr/usagers/politique-dutilisation/ 


\title{
EMPREINTE ET RÉINCARNATION
}

BENOÎT MAUCHAMP

\begin{abstract}
Selon les enseignements bouddhistes, après la mort, nous sommes involontairement réincarnés dans l'existence [...]. Un être ayant atteint l'éveil, un bouddha, peut choisir de son propre gré la prochaine forme de son existence [...]. Les grands maîtres tibétains, les lamas, continuent à se réincarner pour guider leurs disciples et tous les êtres vers l'éveil.
\end{abstract}

(Baratz, 2008; notre traduction) ${ }^{1}$

C'est sur ces intertitres alternant avec les images d'un jeune moine népalais, et quelques autres textes encore qui introduisent ce personnage principal et l'intrigue, que s'amorce le documentaire Unmistaken Child, réalisé par Nati Baratz en 2008. Le réalisateur suit Tenzin Zopa, le moine en question, dans la quête de la réincarnation de son maître Geshe Lama Konchog, récemment décédé.

Little Buddha, une fiction de Bertolucci projetée sur les écrans en 1993, s'ouvre elle aussi, de manière plus romancée et stylisée, sur la notion de réincarnation, au travers d'un conte illustré, lu par Lama Norbu à une classe d'enfants moines dans un monastère du Bhoutan. Il raconte l'histoire d'un prêtre hindou qui allait sacrifier une chèvre quand celle-ci se mit à rire parce qu'enfin, après 999 vies, elle serait réincarnée en être humain. Lui parvient pendant sa leçon un télégramme dont il prend aussitôt connaissance pour finalement murmurer: "Voilà neuf ans que j'attends de recevoir cela...» (notre traduction) ${ }^{2}$. Peut-être a-t-on enfin trouvé la réincarnation de son ancien maitre, Lama Dorje.

Une tradition, née au Tibet au XIII siècle, consiste à reconnaître les réincarnations de ces êtres éveillés, ou tulkus, qui peuvent choisir de renaître pour aider et guider les croyants.

Le but véritable de cette tradition est d'assurer que la mémoire de sagesse des maîtres ayant atteint la réalisation ne se perd pas. Ce qui caractérise la vie d'une incarnation, c'est que sa nature originelle

- la mémoire de sagesse dont il ou elle a hérité - s'éveille à travers son éducation; c'est là le signe indubitable de son authenticité. (Sogyal Rinpoché, 2003: 198-199)

Vers la fin du documentaire de Nati Baratz, l'enfant face à ses deux portraits photographiques, l'un de lui-même (entendons l'enfant), l'autre du maître réincarné, peut donc dire sans encombre ni doute, en pointant du doigt chacune des deux 
images, qu'il est l'un, et qu'il est l'autre également. C'est-à-dire, selon le concept bouddhiste du nonego, ni le même ni l'autre, ou d'après la notion d'impermanence, ni vraiment identique, ni tout à fait différent. Le corps de l'enfant renferme en lui-même l'esprit d'un autre sans qu'ils soient confondus... ni que la personne ne devienne schizophrène!

Ainsi, un enfant pourra-t-il être reconnu en tant que le support corporel de la conscience d'un être éveillé, et ce, tout en demeurant lui-même. Il s'agit donc là de la transcendance d'une conscience contenue dans l'immanence d'un corps, quelque chose qui dépasse l'être mais qui, en même temps, lui est intrinsèque.

La notion d'«empreinte», proposée comme nouveau principe de pertinence sémiotique par Jacques Fontanille dans Soma et Séma, recouvre une dualité tout aussi surprenante et similaire, puisqu'elle est à la fois partie intégrante du signe et qu'elle renvoie à un au-dehors. En conclusion de son ouvrage sur les figures du corps, le sémioticien définit le principe de l'empreinte comme

[...] celui de l'inscription des formes signifiantes dans un substrat matériel; selon ce principe, il n'y a de signification observable que si des corps gardent les traces d'interactions avec d'autres corps, et si les corps «énonçants» parviennent à désenfouir les contenus et les représentations associés aux expériences d'interactions antérieures. (2004: 264)

Une remarque s'impose ici. Dans le cadre des films considérés, les corps énonçants qui désenfouissent les contenus, ou en d'autres termes reconnaissent le tulku pour ce qu'il est, sont les moines tibétains. C'est leur quête donc qui sera considérée dans les derniers temps du développement, plutôt que le modus operandi de l'empreinte filmique. Il ne sera donc pas question d'analyser l'inscription du corps de l'enfant - forme signifiante - sur le support visuel - substrat matériel. Par contre, l'inscription de la conscience du lama décédé dans le corps de l'enfant - devenant dès lors le substrat matériel -, c'est-à-dire le phénomène de réincarnation, sera explicitée, tout en étant rapportée à certains éléments d'une sémiotique de l'empreinte. La réflexion porte donc sur cette empreinte de la conscience d'un être réalisé dans un corps appartenant au monde naturel à partir de concepts théoriques et de représentations cinématographiques; ce corps étant considéré, d'une part, comme l'expression d'un lama réincarné et, d'autre part, comme le contenu essentiel des films.

\section{LE MONDE, LES SENS, L'ESPRIT}

Comme le rappelle François Rastier dans un article intitulé «Saussure, la pensée indienne et la critique de l'ontologie" (2002), l'auteur du Cours de linguistique générale, en élaborant sa théorie du signe, a éprouvé le besoin d'évacuer la notion de référent, au contraire de la conception peircienne du signe. Évaluant les conséquences de la rupture de Saussure avec la tradition ontologique occidentale, François Rastier conclut «la fin de la représentation» et cite le linguiste d'après qui «il n'y a aucune raison d'attendre que les termes [d'une langue] s'appliquent complètement ou même très incomplètement à des objets matériels ou autres» (Saussure, 2002: 65). Pourtant, pour qui s'intéresse à une sémiotique de l'empreinte - et Jacques Fontanille évoque lui-même les rapports que cette sémiotique pourrait entretenir avec la question de la référence -, cette position n'est pas tenable. Tout comme le souligne Paul Ricœur à propos du langage qui est transcendant à lui-même (1975: 97), l'empreinte renvoie nécessairement à un au-delà ou un au-dehors du signe, c'est-à-dire à un référent ayant pu imprimer sa marque sur le système sémiotique considéré.

Il n'empêche que la référentialité est souvent demeurée une question qui ne concerne pas la sémiotique dans la mesure où elle est appréhendée comme extérieure au signe, et qu'il n'y pas de sens a priori en dehors de celui-là.

Les sémioticiens rejettent le concept de référent (l'univers

extralinguistique). Ils considèrent le «monde naturel» comme

une sémiotique dans la mesure où, en tant que plan de

l'expression, il est informé par l'homme et érigé en signification.

[...] Il reste toutefois que cette «information» du monde

naturel demande qu'on examine les conditions d'émergence $d u$ sens à partir du sensible. (Bertrand, 2000: 79)

Et c'est précisément l'orientation qu'ont prise les recherches sémiotiques de ces dernières années, 
portant sur l'esthésie, la poly-sensorialité, et notamment le corps sensible.

Ainsi, en dépit de la réflexion saussurienne, et par nécessité aussi puisque c'est de réincarnation qu'il est ici question, voit-on resurgir le principe du corpssujet, qui occupe l'espace physique tout autant qu'il lui donne sens. En cela, la sémiotique s'accorde avec la pensée bouddhiste, à une nuance près néanmoins, et de taille: tandis que l'accent en sémiotique est mis sur la sensorialité, et donc sur le corps, le bouddhisme condamne les effets néfastes de celle-ci qu'il conçoit comme étant à l'origine de la production d'un monde illusoire que les hommes prennent pour véritable.

Le corps n'est qu'une enveloppe périssable, d'ailleurs désignée par le terme lü en tibétain, «ce qu'on laisse derrière soi» et qui «rappelle que nous ne sommes que des voyageurs, ayant trouvé un refuge temporaire dans cette vie et dans ce corps" (Sogyal Rinpoché, 2003: 60). Trompés par leurs perceptions qu'ils prennent pour vraies quand elles ne sont en fait que conditionnées par leur karma, les êtres sensibles nourrissent cette croyance que rien n'est réel en dehors de ce à quoi s'attachent leurs sens ordinaires. Dès lors, comme l'écrit un maître tibétain du XVIII ${ }^{e}$ siècle, Jigmé Lingpa, cité par Sogyal Rinpoché, «hypnotisés par l'infinie variété des perceptions, les êtres errent et se perdent sans fin dans le cercle vicieux du samsāra» (ibid.: 61). Une preuve de ces perceptions erronées, note l'auteur, réside dans le simple fait que la saisie sensible d'une même chose peut considérablement différer d'un être à un autre. Et pourtant, nous nous y accrochons, créant en cela autour de nous un environnement de dépendances toujours plus fortes, à commencer par la plus puissante qui est celle de l'attachement à soi et de la constitution de l'ego, une «fausse identité, adoptée par ignorance" (ibid.: 229).

C'est peut-être le concept bouddhiste du nonego qui a amené Saussure à critiquer l'ontologie et postuler implicitement, comme le suggère Rastier, "l'étrangeté à soi» en tant que "condition de la connaissance» (2002: 129). De même, sa négligence du référent pourrait relever de la tradition du yogācāra, une branche spécifique du bouddhisme selon laquelle «il n'existe pas d'arrière-monde et tous les phénomènes que nous ressentons sont de simples perceptions sans plus, surgies de l'esprit qui les prend pour référents» (Cornu, 2009: 26).

Par sa pratique méditative qui promeut une présence à soi et un état de non-dualité, le pratiquant (désigné par le terme nangpa en tibétain, qui signifie «tourné vers l'intérieur») doit donc chercher «à dé-solidifier ces fausses perceptions étroitement liées et interdépendantes, qui nous ont fait tomber dans le piège illusoire que nous avons créé de toutes pièces", entendons le monde naturel (Sogyal Rinpoché, 2003: 505). Ainsi, sur le chemin de l'éveil, le moine percevra-t-il son potentiel de bouddhéité. La méditation est le véhicule de la transcendance vers la véritable nature de l'esprit, et «incarner le transcendant est notre raison d'être en ce monde" (ibid.: 168).

Le bouddhisme tibétain distingue en effet deux types d'esprits, la nature de l'esprit d'abord, appelée rigpa, et l'esprit ordinaire ensuite, ou sem, qui rappellent respectivement les concepts peirciens de priméité et de secondéité. Au même titre que la priméité, unité totale et englobante, sans limites ni parties, rigpa est la

$$
\begin{aligned}
& \text { [...] conscience claire, primordiale, pure, originelle, à la fois } \\
& \text { intelligence, discernement, rayonnement et éveil constant. On } \\
& \text { pourrait dire qu'elle est la connaissance de la connaissance } \\
& \text { elle-même. (Ibid.: 106) }
\end{aligned}
$$

Au contraire, la secondéité, en concevant l'être de manière relative à autre chose introduit dans le monde une dualité - dualité qui s'apparente d'ailleurs à la phénoménologie en tant que projection d'une conscience subjective sur les objets du monde. La secondéité présente par conséquent quelques rapports avec le sem,

[...] l'esprit discursif, dualiste, l'esprit «qui pense», qui ne peut fonctionner qu'en relation avec un point de référence extérieur projeté par lui et faussement perçu. (Ibid.: 105)

Rigpa est l'essence même, présente en toute chose et englobant le tout, et par conséquent l'état dans lequel les concepts d'immanence ou de transcendance ne sont pas valides. Sem, au contraire, est ce par quoi la transcendance peut advenir, ce par quoi rigpa peut 
se découvrir. Atteindre l'état d'éveil, c'est pénétrer rigpa, passer du subjectif à l'objectif, au moyen de la désolidarisation de l'esprit ordinaire et des facultés sensorielles, émotionnelles et intellectuelles du corps mental, qui opacifient la nature véritable et fondamentale de l'esprit, c'est-à-dire l'accès au potentiel de bouddhéité présent en chaque être. Du point de vue bouddhiste, le monde naturel est une projection de nos sens gouvernés par notre conscience individuelle qui prend cette projection pour vraie, au même titre que l'espace du film, à un autre degré et dans une perspective référentielle, est le résultat de l'empreinte du monde naturel sur son support, lui aussi souvent faussement pris pour vrai.

Ce développement hybride rapproche sans doute grossièrement des considérations sémiotiques et bouddhistes; néanmoins, la présentation rapide des trois notions de monde naturel, de corps sensible et d'esprit prend tout son sens dans ce qui suit, en ce qu'elle permet une meilleure appréhension du processus de réincarnation, et de sa similitude avec une sémiotique de l'empreinte.

\section{BARDOS, KHAYAS ET SIGNIFICATION}

Le célèbre Livre des morts tibétain, aussi connu sous le nom de Bardo Thödol - et attribué à Padmasambhava, fameux maître bouddhiste du VIII ${ }^{e}$ siècle -, envisage la vie et la mort comme un cycle continu de réalités transitoires constamment changeantes, appelées bardos. Ce terme désigne un état intermédiaire, un passage qui offre une possibilité d'éveil accru. Ils sont au nombre de quatre et se déclinent ainsi: «le bardo naturel de cette vie; le bardo douloureux du moment de la mort; le bardo lumineux de la dharmatā et le bardo karmique du devenir» (Sogyal Rinpoché, 2003: 45). Pour qui cherche à comprendre le phénomène de réincarnation, les trois derniers stades apparaissent cruciaux en ce qu'ils constituent:

[...] les trois phases d'un processus de manifestation graduelle de l'esprit: partant de son état le plus pur - celui de la nature essentielle de l'esprit -, en passant par la lumière et l'énergie - le rayonnement de la nature de l'esprit -, pour arriver, par une cristallisation progressive, à une forme mentale. Ce qui se révèle avec une telle clarté dans le bardo du moment précédant la mort, dans le bardo de la dharmatā et celui du devenir, nous montrent les enseignements, est un processus triple: tout d'abord, une dissolution qui conduit à un dévoilement; ensuite, un rayonnement spontané; enfin, une cristallisation et une manifestation. (Ibid.: 608-609)

Au moment de la mort, le corps sensible se désagrège et avec lui le monde naturel et illusoire également, pour laisser jaillir l'espace d'un instant la lumière vive et fondamentale de la nature de l'esprit. Le détachement que les moines expérimentent durant leur pratique méditative se produit donc naturellement. Inversement, lors du bardo du devenir, l'esprit ordinaire reparaît et, conditionné par son karma passé, est conduit à s'attacher à ce qu'il prend pour des réalités tangibles, mais qui proviennent de ses expériences illusoires.

En définitive, c'est le désir intense de l'esprit d'habiter dans un monde particulier qui nous pousse à nous réincarner, et c'est sa tendance à solidifier et à s'attacher qui trouve son expression ultime dans une renaissance physique.

(Ibid.: 533)

Poussant plus avant sa réflexion sur le triple processus des bardos, Sogyal Rinpoché propose une autre manière de comprendre cette triade, «en observant ce qui est révélé à chacune des phases de la mort» (ibid.: 609) - l'expression, soulignée par l'auteur lui-même, rappelle le principe de désenfouissement évoqué en introduction pour définir l'empreinte. Ainsi rapproche-t-il les bardos de la mort, de la dharmatā et du devenir, des trois kayas, terme sanscrit qui signifie littéralement le "corps", et qui revêt un sens particulier encore dans la perspective d'une sémiotique de l'empreinte, puisqu'elle s'intéresse précisément à la constitution des choses en corpsactants.

1. La nature absolue, dévoilée au moment de la mort dans la Luminosité fondamentale, est appelée dharmākaya; c'est la dimension de la vérité "vide», non conditionnée, dans laquelle ni l'illusion, ni l'ignorance, ni le moindre concept n'ont jamais pénétré.

2. Le rayonnement intrinsèque de l'énergie et de la lumière, qui se déploie spontanément dans le bardo de la dharmatā, est appelé sambhogakāya; c'est la dimension d'une complète 
félicité, le champ d'une plénitude et d'une richesse totale, au-delà de toutes les limitations dualistes, au-delà de l'espace et du temps.

3. La sphère dans laquelle s'opère la cristallisation en une forme et que révèle le bardo du devenir est appelée nirmānakāya; c'est la dimension de la manifestation incessante. (Ibid.: 609-610)

L'expression nirmānakāya est l'équivalent sanscrit du terme tulku; sa traduction directe serait «corps d'apparition" ou "corps émané», à comprendre finalement comme la manifestation, réapparaissant sans cesse dans l'univers physique, de l'énergie de la compassion et de l'éveil.

Poursuivant sa pensée, Sogyal Rinpoché remarque que plusieurs éléments de la vie s'apparentent aux phases des bardos et des trois kayas: le sommeil et les rêves, les expériences de la vie quotidienne, la formation de nos émotions et de nos pensées, et plus particulièrement en ce qui concerne le propos, le processus de la créativité humaine, qu'elle soit scientifique ou artistique, conçue comme:

[...] manifestation en tant que forme du monde intérieur de

l'humanité. [...] Cette manifestation individuelle de créativité [...] jaillit d'une source mystérieuse d'inspiration et est convertie en forme par l'intermédiaire d'une énergie dont le rôle est de traduire et communiquer. (Ibid.: 622-623)

Usant de ces métaphores récurrentes dans la pensée orientale, l'auteur, pour illustrer son propos, compare l'œuvre d'art à la clarté lunaire, qui ne brille pas d'elle-même, mais trouve sa source dans la lumière du soleil caché. Dès lors, le processus créatif résulte de la conversion du dharmakāya en nirmānakāya par l'intermédiaire du sambhogakāya:

La véritable signification de l'expression artistique inspirée ne réside-t-elle pas dans le fait qu'elle est apparentée au champ du sambhogakāya, cette dimension d'énergie incessante, [...] ce rayonnement qui transmet, traduit et communique la pureté et le sens infini de l'absolu à ce qui est fini et relatif - qui, en d'autres termes, conduit du dharmakāya au nirmānakāya?

(Ibid.: 625)

S'appuyant sur les réflexions scientifiques du physicien David Bohm qui insistent sur l'implication mutuelle du sens, de l'énergie et de la matière, Sogyal Rinpoché suggère enfin que sens, énergie et matière ont entre eux un rapport similaire à celui qui existe entre les trois kayas. Le sens absolu est d'une certaine manière analogue au dharmakāya, "totalité inconditionnée»; l'énergie a des affinités avec le sambhogakāya, «jaillissement spontané»; et la matière présente des ressemblances avec le nirmānakāya.

\section{L'EMPREINTE (1):}

INTERACTION ENTRE ÉNERGIE ET MATIÈRE

$\mathrm{Si}$, pour conclure, Sogyal Rinpoché plaide pour le rapprochement des sciences pures et de la mystique, il semble tout aussi intéressant et justifié de les rapporter aux principes d'une sémiotique de l'empreinte. De manière réciproque d'ailleurs, Jacques Fontanille fait lui-même référence aux différentes cosmogonies qui toutes, indépendamment de leur horizon culturel, invoquent «des formes du déploiement de l'énergie [...] port[ant] sur des structures matérielles» (2004: 262-263). Au même titre donc que la signification pure (ou dharmakāya) se manifeste dans le monde naturel (sous la forme du nirmānakāya) par le biais d'une énergie (ou sambhogakāya),

[...] l'empreinte, ce principe qui permet de saisir la sémiotisation du monde par des corps, est donc elle-même soumise, en quelque manière, aux interactions entre matière et énergie, c'est-à-dire à la syntaxe figurative. (Ibid.: 266)

Il convient par conséquent, pour toute étude s'intéressant au pouvoir signifiant de l'empreinte, de considérer à la fois le caractère corporel de l'objet matériel qui reçoit l'inscription - en l'occurrence le corps physique de l'esprit ordinaire - et celui de l'énergie motrice à la base du geste d'énonciation, c'est-à-dire de cette inscription. D'ailleurs, cette remarque, qui conclut le travail de Jacques Fontanille sur les figures du corps, rappelle le dernier chapitre du livre dans lequel l'auteur s'intéresse à la patine (résultat d'une somme d'usages particuliers appliqués à un objet qui en recueille les traces) et à la conversion des choses en corps-objets.

L'empreinte est le strict équivalent d'une trace énonciative

d'une inscription, dans l'énoncé même de l'objet, de la chaîne répétitive des actes dont il a été l'instrument, le lieu ou 
l'occasion, et tout comme la langue, cet usage en continu le modifie en permanence, mais en surface. (Ibid.: 248)

De manière similaire à cette inscription continuelle des actes sur l'objet, le karma de l'esprit ordinaire possède une mémoire de stockage de ses interactions antérieures avec d'autres corps-objets. S'appuyant sur le commentaire d'un éminent maître tibétain du XIVe siècle, Longchenpa, Sogyal Rinpoché rappelle ainsi que la base de l'esprit ordinaire est

[...] le fondement de tous les karmas et de toutes les "traces" du samsära [...]. Il fonctionne comme un entrepôt dans lequel toutes les empreintes de nos actions passées, causées par les émotions négatives, sont stockées comme des graines.

(2003: 219-220)

C'est pourquoi, lors du bardo du devenir, le désir d'un corps matériel est gouverné par les habitudes passées du karma. Si, dans l'immense majorité des cas, trop fortement induits en erreur par les émotions et les désirs de notre esprit ordinaire, nous n'avons aucun choix véritable de notre prochaine naissance, les lamas ayant maîtrisé la loi du karma peuvent, en revanche, orienter leur prochaine naissance et imprimer leur marque dans le corps le plus approprié à leur mission de guide spirituel. Un cas très particulier est mis en scène dans Little Buddha, qui d'ailleurs constitue le ressort de l'intrigue: alors qu'au début du film Lama Norbu se rend à Seattle pour rencontrer Jesse, qui a été pressenti par un moine (d'ailleurs joué par Sogyal Rinpoché) comme la réincarnation de Lama Dorje, il apprend vers la fin de son séjour qu'un autre enfant a été repéré à Katmandou. Une fois au Népal où il rencontre le jeune Raju, l'information lui parvient alors qu'un troisième enfant encore, habitant un village en Inde, pourrait également être la manifestation de son maître décédé il y a une dizaine d'années. D'abord désorienté, Lama Norbu finit par s'amuser de la situation, y voyant là un trait particulier de l'humour légendaire de son maître, qui a donc délibérément choisi de se réincarner en trois individus - peut-être est-ce la raison pour laquelle alors la nouvelle de cette réincarnation apparaît si tardive, les tulkus étant dans la plupart des cas identifiés entre deux et sept ans...
Après le passage en Inde donc, tous les personnages (Lama Norbu et son assistant, Jesse accompagné de son père, Raju de Katmandou et Gita l'Indienne) se rendent dans un monastère du Bouthan afin de procéder aux vérifications qui s'imposent et qui seront détaillées plus bas.

L'interaction entre énergie et matière suppose par ailleurs un principe de contiguité, sur la base duquel peut s'établir un autre parallèle entre empreinte et réincarnation. Dans le même chapitre ayant trait à la patine, Jacques Fontanille oppose celle-ci à l'objet ergonomique: tandis que ce dernier reçoit pour empreinte un programme idéal fondé sur une analogie, la patine est le résultat au contraire d'une adaptation progressive de l'objet à l'usage, de la matière à l'énergie en d'autres termes, qui se fait à force de répétition et par contiguité.

Les vies successives d'une série de renaissances ne sont pas semblables aux perles d'un collier maintenues ensemble par un fil, "l'âme», qui passerait à travers les perles; elles ressemblent à des dés empilés l'un sur l'autre. Chaque dé est séparé, mais il soutient celui qui est posé sur lui et avec lequel il a un lien fonctionnel. Les dés ne sont pas reliés par l'identité, mais par la conditionnalité. (Schumann, 1989: 55)

Si cette conditionnalité est précisément le résultat des empreintes successives accumulées dans le karma durant le bardo naturel de la vie, ce karma néanmoins ne saurait être assimilé à l'âme, car le bouddhisme ne croit pas en une telle entité, indépendante ou immuable, survivant à la mort du corps. Au même titre que l'objet - continuellement altéré par les usages successifs qu'il subit - ne saurait être identique à luimême, le karma, fonctionnant comme un réservoir, est en permanence modifié par les émotions ou les actes qui l'animent. En outre, tout comme les usages de l'objet patiné sont répétés mais discontinus, le cycle des renaissances l'est également, suspendu durant les périodes de transition que constituent les bardos de la mort, de la dharmatā et du devenir. Cette relative discontinuité n'enlève cependant rien au principe de contiguité si bien imagé par les dés empilés; le contact d'une vie à une autre s'opérant précisément durant le bardo du devenir, lorsque l'esprit ordinaire se re-matérialise. Enfin, il faut encore spécifier que, s'il 
y a contiguité d'une vie à l'autre, il y a aussi contiguïté entre l'esprit et le corps, tous deux conçus par la philosophie bouddhiste comme des substances qui, si elles s'influencent l'une l'autre, ne présentent en aucun cas un rapport de causalité comme l'indique le Dalaï-Lama lors d'un enseignement donné à New York en octobre 1991:

Dans le cas de l'esprit et du corps, bien que l'un puisse affecter l'autre, l'un ne peut pas devenir la substance de l'autre... Bien que l'esprit et la matière dépendent l'un de l'autre, l'un ne peut être la cause substantielle de l'autre. C'est sur cette base que le bouddhisme accepte la notion de renaissance.

(Sogyal Rinpoché, 2003: 183)

Cette notion de contiguité est essentielle dans la mesure où, d'une part, elle rappelle la caractéristique indicielle de l'empreinte selon la typologie peircienne des signes (un contact a [eu] lieu entre le signe et son référent, entre l'enfant et la conscience réincarnée), et, d'autre part, elle supporte l'idée que «l'empreinte assure la présence sensible des systèmes signifiants» (Fontanille, 2004: 265).

\section{L'EMPREINTE (2):}

\section{PRÉSENTIFICATION D'UNE ABSENCE}

L'interaction entre matière et énergie était l'une des deux hypothèses avancées dans Soma et Séma pour comprendre la syntaxe figurative, la seconde étant celle de la présence:

Si la figurativité se caractérise par l'existence de correspondances entre un univers sémiotique et le monde naturel, mais de correspondances reposant sur des équivalences perceptives, dépendantes de la position de l'observateur dans le monde naturel, alors il faut chercher le principe organisateur de la syntaxe figurative du côté de la présence perçue des figures. (Ibid.: 262)

Avant d'observer comment cette présence d'une conscience éclairée est perçue par les protagonistes des films évoqués en introduction, il convient de les décrire un peu plus spécifiquement. Unmistaken Child et Little Buddha retracent respectivement les quêtes de Tenzin Zopa (le jeune moine du documentaire) et de Lama Norbu, chacun recherchant la réincarnation de son maître décédé. Comme cela a déjà été remarqué par ailleurs, il n'est pas question ici de trop insister sur l'aspect formel de ces réalisations. Le rapprochement des deux films a été fait pour des raisons de contenus essentiellement, la fiction présentant une certaine vraisemblance (l'intrigue est contemporaine et la tradition représentée réelle).

Le caractère particulier d'une réincarnation triple, détaillée précédemment, motive le parcours narratif de Little Buddha, de Seattle au Bouthan, en passant par le Népal et l'Inde. La recherche du tulku ici semble quelque peu biaisée dans la mesure où l'enfant américain, Jesse, personnage principal du film, est trouvé d'emblée, au même titre que ses deux amis. Il n'y a de quête dès lors que dans le processus de vérification de ces réincarnations supposées, qui, néanmoins, n'occupe que la dernière demi-heure du film. En outre, tandis que Little Buddha se déroule sur une période de deux mois, la recherche de Tenzin Zopa, personnage principal du documentaire, s'étale sur presque cinq années, depuis les funérailles de Geshe Lama Konchog en octobre 2001, jusqu'à l'arrivée au monastère, en mars 2006, du petit Rinpoché (titre honorifique réservé à un lama réincarné) rebaptisé Tenzin Ngodrup par le Dalaï-Lama lui-même. Entre-temps ont lieu en 2002 les interprétations et calculs astrologiques aiguillant la recherche du moine, plusieurs mois durant, de village en village, dans une vallée de l'Himalaya, et les processus de vérification et confirmation qui, quoique ponctuels, s'étalent sur trois années.

À l'image de l'ouverture de Little Buddha sur un intertexte (le conte illustré), la trame première de la fiction alterne très fréquemment (grossièrement par séquence de cinq à dix minutes) avec l'histoire du prince Siddhārta Gautama jusqu'à l'éveil de Bouddha, qui constitue l'acmé du film (les deux trames se confondent d'ailleurs durant cette séquence: les trois enfants assistent littéralement à cette scène de l'éveil). On peut s'interroger sur la justification, autre qu'esthétique, de ce récit mythique qui ne sert pas au premier abord la trame réaliste de la fiction, et éventuellement lui supposer une signification symbolique en tant qu'initiation éducative de Jesse au bouddhisme (ce récit secondaire s'insère en effet dans l'intrigue du fait que les moines tibétains offrent à 
l'enfant un livre relatant l'histoire du Bouddha). Car afin de réveiller le potentiel énorme de compassion, de bienveillance et de sagesse que possèdent les tulkus, ceux-là quand ils sont enfants reçoivent un enseignement particulier. C'est ce qu'explique Tenzin Zopa aux parents du petit Rinpoché dans la dernière phase du documentaire, qui comporte dès lors quelques scènes tragiques du fait de la séparation de l'enfant d'avec sa famille.

La prise en charge de l'enfant par le monastère est donc destinée à désenfouir ce qu'il possède en lui, en tant que support tangible de l'empreinte d'un être réalisé, et qui est une conscience ayant atteint l'éveil et pénétré rigpa, au terme d'un très long processus de morts et de renaissances. La conscience éclairée s'étant inscrite dans le corps de l'enfant, mais n'ayant pas encore été révélée, il s'agira donc pour les maîtres de l'enfant de parvenir à la "présentification d'une absence» et de réactualiser, en somme, «la présence maintenue et potentielle de l'origine», ou dharmakāya.

L'empreinte est une trace qui [...] suscite tout un programme interprétatif: dès lors, une empreinte ne vaut que si elle est confirmée par d'autres indices, si elle corrobore des hypothèses, si elle permet de construire des inférences créatives.

(Fontanille, 2004: 254)

Avant de pouvoir travailler au réveil du potentiel contenu dans les jeunes tulkus, les moines, en quête des réincarnations de leurs maittres, doivent donc être à même de percevoir, dans l'être qu'ils ont pressenti, la présence sensible de cette conscience subtile. Aussi sont-ils en mesure $a$ priori d'interpréter plusieurs indices confirmant que l'enfant est bien la manifestation authentique de cette conscience.

Le déchiffrement de ces indices est varié, mais leurs formes les plus populaires passent par le rêve, d'une part, et la reconnaissance par l'enfant des objets rituels lui ayant appartenu dans sa précédente existence, d'autre part.

Lorsqu'un maître ayant atteint la réalisation meurt, il ou elle laisse parfois des indications très précises sur le lieu de sa renaissance. Il se peut alors qu'un de ses disciples ou amis spirituels très proches ait une vision ou un rêve annonçant sa renaissance imminente. (Sogyal Rinpoché, 2003: 198)
Des rêves sont effectivement évoqués dans Little Buddha et Unmistaken Child. Ceux de Kenpo Tenzin, qui accueille Lama Norbu à Seattle où Lama Dorje est décédé, ont commencé dès un mois après la disparition du maitre. Pendant longtemps, le maître lui apparaissait dans la rue et le guidait vers un terrain vague qu'il a finalement retrouvé, et où a été bâtie récemment une maison. Kenpo Tenzin continue son récit jusqu'à ce que, finalement, la petite délégation de moines tibétains sonne à la porte de cette maison moderne et soit reçue dans le salon, où alors, mystérieusement, Jesse apparaît avec un masque recouvrant son visage, comme pour symboliser la présence cachée du maître réincarné dans l'enveloppe corporelle.

En revanche, le rêve que raconte Tenzin Zopa devant la caméra, quoique attendrissant et rempli d'espoir dans la mesure où il signifie la très probable réincarnation de son maître, n'indique rien d'aussi clair et direct que les visions précédentes:

La nuit dernière j'ai fait un rêve... Le corps sacré de Geshe

Lama Konchog et sa dépouille gisant au-dehors... quand un petit garçon sortit de la dépouille. Il était tout potelé et tout petit, vous savez. Et puis il m'appelait: «Tenzin Zopa...

Tenzin Zopa...donne-moi la main». Alors, je lui tendis la main. Vous savez, l'émotion dans le rêve était tellement joyeuse que, tout d'un coup, cette joie me réveilla.

(Baratz, 2008; notre traduction) ${ }^{3}$

Contrairement à la fiction de Bertolucci qui passe rapidement sur la quête au sens propre de la nouvelle manifestation de l'être éveillé, le documentaire révèle d'autres indications bien plus précises quant à la direction dans laquelle orienter les recherches de la réincarnation de Geshe Lama Konchog: la direction de la fumée lors de sa crémation ou l'empreinte de pas laissée dans les cendres avant qu'elles ne soient recueillies sont aussi des indices à interpréter. Mais ce qui précipite finalement Tenzin Zopa dans la vallée himalayenne où trouver son maître, ce sont les résultats des calculs astrologiques réalisés par un autre moine, sur la base notamment d'un dessin qu'il a réalisé lui-même, des environs du monastère de Kopan. Avec une probabilité de $95 \%$ donc, le nom du lieu où rechercher le lama aurait pour initiales $\mathrm{T}$ et 
S - nul doute qu'il s'agit de la Tsum Valley où Geshe a fait une retraite de vingt-six ans et où Tenzin est né de surcroît -, et le nom du père de la réincarnation devrait commencer par la lettre A...

Une fois l'enfant trouvé, l'étape suivante consiste à s'assurer s'il s'agit bien du tulku recherché, notamment au moyen - le seul suggéré par les films - de la reconnaissance des objets rituels lui ayant appartenu dans sa vie antérieure. Plusieurs types d'objets similaires sont donc exposés à l'enfant (cloches, tambours, chapelets et boîtes, dans le cas du documentaire), mais il n'y en a qu'un seul dans la fiction (des chapeaux). Ce rite semble être généralement décisif pour l'authentification du tulku. Néanmoins, devant la situation particulière à laquelle Lama Norbu fait face - les trois enfants choisissant le bon chapeau -, celui-ci aura recours à un chaman.

Cet examen est intéressant dans la mesure où il renvoie à la patine dont il était question plus haut, les objets rituels ayant précédemment reçu la marque de leur usage par le lama décédé et recherché. Cependant que cette empreinte sur la surface de l'objet est superficielle et ne provint pas de son usage par un individu particulier - car en général l'empreinte de la patine est le résultat d'un emploi collectif et donc impersonnel -, l'enfant pressenti reconnaît ce qui était sien et, par là, est à son tour reconnu par les moines comme porteur de la marque, intérieure cette fois, de la conscience d'un être éveillé.

La sémiotique de l'empreinte prête attention au modus operandi de la production textuelle, tout autant qu'à celui de l'interprétation, car elle fait l'hypothèse que l'interprétation est une expérience qui consiste à retrouver les formes d'une autre expérience, dont il ne reste que l'empreinte.

(Fontanille, 2004: 265)

Ce processus fonctionne par exemple dans le cas des objets rituels, mais à un autre niveau: la trace énonciative de la conscience réincarnée s'est inscrite au-dedans du corps et non sur son enveloppe. En toute logique, les moines en quête de leurs maîtres, les interprétants, devraient donc être à même de désenfouir cette marque ( retrouver les formes d'une autre expérience»); néanmoins, ils ne le font au final que par un moyen détourné qui consiste à mettre l'enfant lui-même (porteur potentiel de cette "autre expérience») en situation de déchiffrement ou d'interprétation d'un texte second, c'est-à-dire autre que celui de sa personne: celui des objets rituels. Cette prothèse employée par les moines laisse par conséquent une question en suspens, et qui peutêtre relève de l'irreprésentable en tant qu'elle n'est sans doute pas inscrite dans le monde naturel tel que nous le percevons: les moines peuvent-ils identifier directement la réincarnation d'un maitre, sans avoir recours finalement à ce stratagème de l'objet reconnu? Sogyal Rinpoché apporte un élément de réponse en affirmant «qu'il n'y a pas, qu'il n'y a jamais eu et qu'il n'y aura jamais de séparation entre lui-même et le maître [...] car le maître est un avec la nature de votre esprit» (2003: 100-101). Et il relate l'histoire de Lama Tseten qu'il a vu mourir et à qui on a demandé au seuil de sa mort s'il souhaitait la présence physique de son maître, ce à quoi le lama répondit: «avec le maittre, la distance n'existe pas». Une autre forme de contiguilté peut-être.

Au fil de sa réflexion, Sogyal Rinpoché observe certaines analogies entre les bardos (transitions) prenant cours au moment de la mort et les trois kayas (corps) d'une part, puis entre ces kayas et les processus artistique et de découverte scientifique d'autre part. Il établit finalement un parallèle entre mystique et physique sur la base des travaux de David Bohm. De la même manière, des similitudes pertinentes existent entre bouddhisme et sémiotique, et, plus particulièrement, pour ce qui concerne le propos, entre le phénomène de la réincarnation et une nouvelle épistémologie sémiotique fondée sur l'empreinte. Les deux hypothèses que rappelle Jacques Fontanille au terme de Soma et Séma, pour rendre compte du principe organisateur d'une syntaxe figurative, sont deux caractéristiques complémentaires de l'empreinte, pleinement applicables à la notion de réincarnation: interaction entre énergie et matière d'une part, qui imprime sur le corpsobjet les traces d'une conscience transcendantale (celle de l'énonciateur ou celle du lama éveillé); présentification d'une absence d'autre part, qui suppose que le corps-objet en question contient en 
son sein cette conscience, dès lors immanente à tout être vivant dans l'absolu. Cependant, qu'il s'agisse de l'enveloppe corporelle ou de la chair de l'objet, en dépit de l'aspect indiciel de la réincarnation qui a été mis en valeur ici, ce phénomène reste relativement, et paradoxalement, imperceptible.

\section{NOTES}

1. "According to Buddhist teachings, after death we are involuntarily reincarnated back into existence [...]. A being who has achieved

enlightment, a buddha, can willingly choose their next life form [...].

The great Tibetan Masters, the Lamas, continue to reincarnate to guide their followers and all beings to enlightment."

2. "I've been waiting for nine years to receive this."

3. "Last night I had a very interesting dream... Geshe Lama Konchog

holy body, and his dead body lying outside... and from Geshe Lama Konchog's dead body, a little boy came out. And he was like quite fattyfatty and quite small, you know. So then he called my name: 'Tenzin Zopa... Tenzin Zopa... give me your hand.' So then I passed my hand. You know the feeling in the dream was so joyous and suddenly with that joy, I was waken up."

\section{RÉFÉRENCES FILMIQUES ET BIBLIOGRAPHIQUES}

BARATZ, N. [2008] : Unmistaken Child, Israël, Samsara Films et Alma Films, 102 minutes, couleur.

BerTOluCCI, B. [1993]: Little Buddha, France, Grande-Bretagne, Italie, Liechtenstein, prod. de J. Thomas, 123 minutes, $35 \mathrm{~mm}$, couleur. BerTRAND, D. [2000]: Précis de sémiotique littéraire, Paris, Nathan. BOHM, D. [1980]: Wholeness and the Implicate Order, Londres, Routledge.

CORNU, P. [2009]: «Introduction», dans Padmasambhava, Le Livre des morts tibétain, Paris, Buchet-Chastel, 19-55.

FonTANille, J. [2004]: Soma et Séma. Figures du corps, Paris, Maisonneuve et Larose.

RASTIER, F. [2002]: «Saussure, la pensée indienne et la critique de l'ontologie", Revue de sémantique et de pragmatique, nº 11, 123-146.

Ricceur, P. [1975]: La Métaphore vive, Paris, Seuil.

SAUSSURE, F. de [2002]: Écrits de linguistique générale, Paris, Gallimard. SCHumann, H. W. [1989] : The Historical Buddha. The Times, Life, and Teachings of the Founder of Buddhism, Londres, Arkana.

SOGYAL RINPOCHÉ [(1993) 2003]: Le Livre tibétain de la vie et de la mort, Paris, Éd. de la Table Ronde. 